OPEN ACCESS

Edited by:

Mika Erik Tapio Sillanpää,

Florida International University,

United States

Reviewed by:

Eric D. van Hullebusch,

Université de Paris, France

Jacek A. Koziel,

lowa State University, United States

*Correspondence:

Armando C. Duarte aduarte@ua.pt

TORCID:

Armando C. Duarte orcid.org/0000-0002-4868-4099

Specialty section

This article was submitted to Environmental Analytical Methods, a section of the journal Frontiers in Environmental Chemistry

Received: 15 April 2020 Accepted: 08 May 2020

Published: 03 June 2020

Citation:

Duarte AC (2020) Specialty Grand Challenges in Environmental Analytical Methods. Front. Environ. Chem. 1:4. doi: 10.3389/fenvc.2020.00004

\section{Specialty Grand Challenges in Environmental Analytical Methods}

\author{
Armando C. Duarte ${ }^{* t}$ \\ Department of Chemistry \& CESAM, University of Aveiro, Aveiro, Portugal
}

Keywords: analytical chemistry, environmental chemistry, environmental analysis, analytical methods, characterisation, sample processing, analytical quality control

\section{INTRODUCTION}

In research fields such as Environmental Chemistry, it becomes fundamental to have methods based on general principles of Analytical Chemistry closely associated with the concept of Fitness for Purpose by adding Quality Control and Quality Assurance protocols. Figure 1 shows the role of Analytical Methods, based on Analytical Chemistry, in the essentially applied field of Environmental Chemistry to produce results with significance in Environmental Analytical Chemistry. The proper use of the links shown in Figure 1, where the whole analytical process is depicted, will lead to results obtained by measurement procedures for measurands in a plethora of matrices of interest with an adequate degree of confidence that can be used both in routine and research laboratories. Figure 1 also shows in detail the complex interrelationships between the interconnected major stages of a method based on Analytical Chemistry but applied to research on Environmental Chemistry Topics, thus resulting in measurements which are the essence when anything chemical in nature is to be known and manageable in research areas associated with Environmental Monitoring and Protection.

Traditionally and academically, the "Problem Definition" is the first step when dealing with issues of environmental significance, but in practice, the different steps of the whole analytical process are intertwined and interact with each other. The different steps cannot be thought of as a sequential mechanism, although for organizational purposes they are often considered as such. For the sake of considering the Specialty Grand Challenges in Environmental Analytical Methods this somewhat sequential and determinist approach will be followed, but researchers should keep in mind a more global and interactive model between the different steps for producing results in Environmental Analytical Chemistry and submitting their work for publication under this Specialty in Environmental Analytical Methods.

\section{PROBLEM DEFINITION: SETTING UP THE BOUNDARIES AND SCOPE OF A CLEAR ENVIRONMENTAL ISSUE}

The main reason why this is considered the first step in Environmental Analytical Methods is that most of the following steps, thinking sequentially, depend and are heavily affected by the environmental issue under study and the limitations taken into consideration for characterizing and assessing such an issue. However, once the environmental issue is clearly defined the development and application of the whole analytical process will necessarily bring back and forward the researcher several times to this step for confirming that 


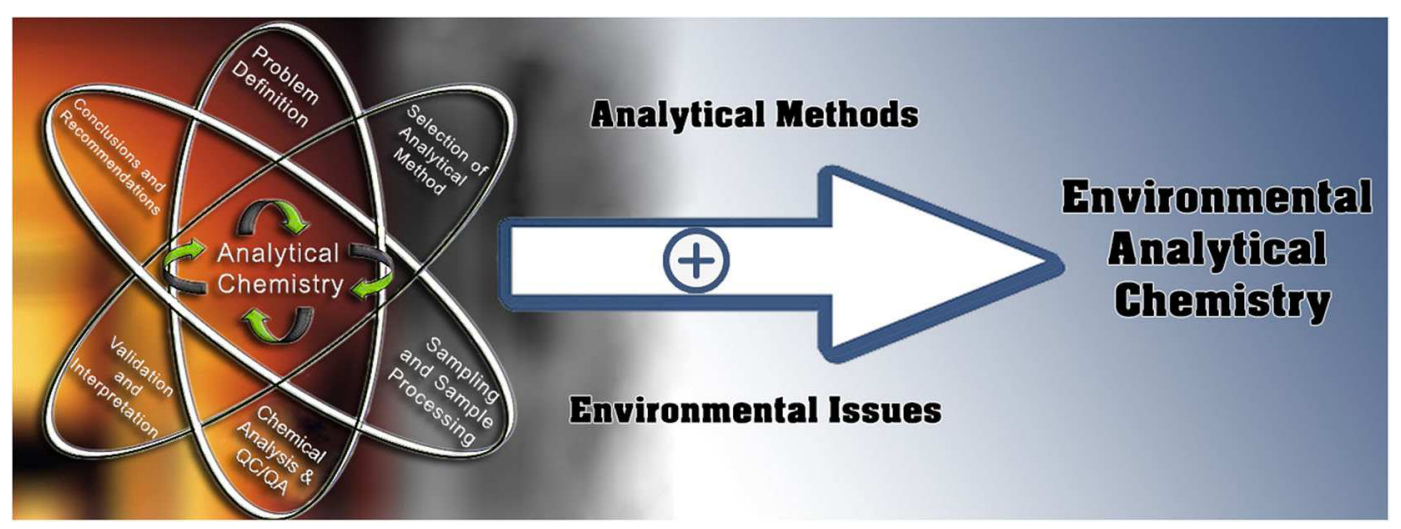

FIGURE 1 | The central role of analytical chemistry when applied to environmentally significant issues for producing results in environmental analytical chemistry.

the initial problem definition is kept unaltered as the analytical process evolves.

\section{SELECTION OF ANALYTICAL METHOD: THE CONCEPT OF FITNESS FOR PURPOSE}

The choice of one or more analytical methods to be used, both for environmental monitoring and for environmental research development, should be based on keeping a broad view over the principle of fitness for purpose. Even for the development of a new analytical method for the application in environmental chemistry issues, this concept has to be taken into consideration by demonstrating clearly what is gained in terms of detection, characterization, and quantification for such an investment of resources.

\section{SAMPLING AND SAMPLE PROCESSING: AN INTERDISCIPLINARY APPROACH RULED BY CHEMISTRY AND STATISTICS}

Although normally the step "sampling and sample processing" of environmental matrices comes together when considering this Specialty of Environmental Analytical Methods, both "sampling" and "sample processing" faces particular difficulties that should be kept in mind and taken into account for the advancement of research and quality of the data produced. Statistics for ruling the sampling strategies and Chemistry for ruling the transformation of the sample into an amenable specimen for analysis represent the two main challenges at this stage of any environmental analytical method. For example, a hot topic nowadays is the characterization of various environmental matrices for assessing "microplastics" (Rocha-Santos and Duarte, 2017), "nanoplastics" (da Costa et al., 2019), and "manufactured nanoparticles" (Hochella et al., 2019) and it is easy to verify that despite the publication of numerous works, one of the great challenges still is sampling and sample processing, not to mention the choice of suitable analytical methods fit for the environmental matrices under study.

\section{CHEMICAL ANALYSIS AND QUALITY CONTROL AND QUALITY ASSURANCE: METROLOGY AND TRACEABILITY}

When performing the chemical analysis, the researcher should make sure that the metrological principles are closely followed and reported. The traceability of the work is only assured when following strictly the methodologies guided by principles of analytical quality control and quality assurance. Although these principles are highlighted here in this step and they are more frequently associated with the laboratory work, they also hold and are of the essence in all the other steps namely, sampling and sample processing. The grand challenge here is to implement protocols both in the field and in laboratory work that guarantee the results can be reproduced anywhere else with a certain degree of confidence.

\section{VALIDATION AND INTERPRETATION: INTERCOMPARISON OF RESULTS AND STUDIES}

The results and associated degree of confidence would allow to compare studies and provide interpretations that could support the development of new studies. The grand challenge here is to provide not only results and the associated estimate of confidence but also a measure of how these results can be used to discuss and to compare with previous works, based on all the supporting information provided on the analytical environmental method, either applied or developed.

\section{CONCLUSIONS AND}

RECOMMENDATIONS: SUPPORT OF ENVIRONMENTAL DECISIONS WHICH COULD LEAD TO NEW ANALYTICAL PROPOSALS AND FOLLOW-UP STUDIES

In any application of Analytical Methods to Environmental Issues, the resulting conclusions and recommendations should 
be the scientific support for taking decisions related to the environmental issues under study. Quite often the results will highlight the limitations of the particular study carried out and it would lead to new analytical proposals and follow-up studies, initiating a new analytical cycle as shown in Figure 1. Most likely either the initial issue has to be rephrased or a completely new problem has to be identified, thus leading to set up new boundaries and scope of a now clearer environmental issue.

\section{WRAP UP AND FINAL CONSIDERATIONS}

Besides the challenges involving the several steps which should be considered in any process of producing data of environmental significance using suitable analytical methods, nowadays there are several specific hot topics which should be highlighted as grand challenges and where any advancements in the environmental analytical methods would be most welcome:

- Sampling, Sample Processing, Separation, Analyte Detection, Quantification with special emphasis in the actual environmental issues associated with microplastics, nanoplastics, and manufactured nanoparticles in environmental matrices

- Assessment of uncertainties in measurements with environmental significance

- Analytical Quality Control and Quality Assurance particularly applied to emerging multidimensional analytical methods, such as omics analytics

- Speciation analysis and related analytical issues in environmental chemistry, such as the analytical basis for assessing the ecotoxicological effects
- Fit-for-purpose methods for key species in environmental matrices mainly when applied in environmental monitoring

- Multidimensional analytical methods for complex environmental matrices with emphasis on the characterization of natural organic matter

- Methodologies for large data sets and extraction of environmental relevant information

- Analytical methods for emerging environmental issues mainly related to global issues such as marine pollution, air quality, and anthropogenic footprints on the planet

- References materials, method validation and chemometrics in environmental analysis.

All studies to be considered as tackling a Specialty Grand Challenge in Environmental Analytical Methods must contribute to the advancement of analytical methodologies and concomitantly to deepen the knowledge into environmental processes.

\section{AUTHOR CONTRIBUTIONS}

The author confirms being the sole contributor of this work and has approved it for publication.

\section{FUNDING}

Thanks are due to CESAM (UIDP/50017/2020+ UIDB/50017/2020), with the financial support from FCT/MCTES through national funds and to project MARSENSE (PTDC/BTA-GES/28770/2017) by national funds (OE) through FCT/MCTES.

\section{REFERENCES}

da Costa, J. P., Reis, V., Paco, A., Costa, M., Duarte, A. C., and Rocha-Santos, T. (2019). Micro(nano)plastics - analytical challenges towards risk evaluation. Trends Anal. Chem. 111, 173-184. doi: 10.1016/j.trac.2018.12.013

Hochella, M. F., Mogk, D. W., Ranville, J., Allen, I. C., Luther, G. W., Marr, L. C., et al. (2019). Natural, incidental, and engineered nanomaterials and their impacts on the Earth system. Science 363:aau8299. doi: $10.1126 /$ science.aau 8299

Rocha-Santos, T.A. P., and Duarte, A. C. (2017). Characterization and Analysis of Microplastics, Comprehensive Analytical Chemistry Series, Volume 75. Amsterdam: Elsevier. doi: 10.1016/bs.coac.2016.10.004
Conflict of Interest: The author declares that the research was conducted in the absence of any commercial or financial relationships that could be construed as a potential conflict of interest.

Copyright $\odot 2020$ Duarte. This is an open-access article distributed under the terms of the Creative Commons Attribution License (CC BY). The use, distribution or reproduction in other forums is permitted, provided the original author(s) and the copyright owner(s) are credited and that the original publication in this journal is cited, in accordance with accepted academic practice. No use, distribution or reproduction is permitted which does not comply with these terms. 